\title{
Nernst Effect and Anomalous Transport in Cuprates: A Preformed-Pair Alternative to the Vortex Scenario
}

\author{
Shina Tan and K. Levin \\ James Franck Institute and Department of Physics, University of Chicago, Chicago, Illinois 60637
}

\begin{abstract}
We address those puzzling experiments in underdoped high $T_{c}$ superconductors which have been associated with normal state "vortices" and show these data can be understood as deriving from preformed pairs with onset temperature $T^{*}>T_{c}$. For uncorrelated bosons in small magnetic fields, and arbitrary $T^{*} / T_{c}$, we present the exact contribution to all transport coefficients. In the overdoped regime our results reduce to those of standard fluctuation theories $\left(T^{*} \approx T_{c}\right)$. Semi-quantitative agreement with Nernst, ac and dc conductivity and diamagnetic measurements is quite reasonable.
\end{abstract}

PACS numbers: $74.40 .+\mathrm{k}, 74.25 . \mathrm{Fy}, 74.72 .-\mathrm{h}$

Keywords: high temperature superconductivity; superconducting fluctuation

\section{INTRODUCTION}

The extent to which the normal state of the high $T_{c}$ superconductors is anomalous has long been debated. The most conclusive evidence for a break-down of Fermi liquid theory has appeared relatively recently via a (pseudo)gap in the fermionic spectrum with onset temperature $T^{*}$. Recent Nernst ${ }^{1.2}$ and ac conductivity ${ }^{3}$ experiments have led to some of the most exotic indications for this pseudogap which, it is claimed, appear in the form of "vortices above $T_{c}$ ". More generally (but not universally) one associates the complex of pseudogap phenomena with some form of precursor superconductivity. While normal state vortices are most closely associated with the well known phase fluctuation scenario $\stackrel{4}{ }$, a primary goal of this paper is to address these same anomalous transport data 2.3 within the alternative pair fluctuation scheme ${ }^{5.6}$. In the process, we present a natural extension of time dependent Ginsburg-Landau (TDGL) theory and associated transport coefficients which addresses higher temperatures $T^{*}$ well outside the usual limited range of applicability near $T_{c}$. It necessarily follows that the bosons appear as quantum rather than classical fields.

The pair fluctuations which we discuss here have as a natural antecedent the Gaussian fluctuations of TDGL. Indeed, when pseudogap effects are weak $\left(T^{*} \approx T_{c}\right)$ TDGL theory has been found to provide a reasonably $\operatorname{good}^{7.8 .9}$ representation of the non-critical fluctuation regime in the high temperature superconductors. In this way the presence of "pre-formed pairs" has been demonstrated, albeit, in the narrow temperature window near $T_{c}$. Two important features of the cuprates suggest that BCS theory is not applicable in that dominant fraction of the phase diagram associated with the pseudogap phase. The anomalously short coherence length $\xi$ in the cuprates combined with the observation of a non-vanishing excitation gap present at the onset of superconductivity has motivated a number of authors 5.6 to contemplate stronger than BCS attraction so that the bosonic degrees of freedom appear at a temperature $T^{*}$ which may be significantly larger than $T_{c}$. Below $T_{c}$ the counterpart of these pre-formed pairs appears as non- condensed bosons. The resulting superconducting state is mid-way between BCS and Bose Einstein condensation (BEC) $)^{5.11 .12}$. One can, moreover, establish 13 that this BCS-BEC crossover approach is closely related to (Hartree-approximated) TDGL theory.

Bosonic degrees of freedom are, in fact, stabilized by going beyond the usual ${ }^{7.9}$ Gaussian approximation. When their interactions are included at the self consistent Hartree leve ${ }^{13.14}$ a fermionic pseudogap necessarily appears; as a result of this depletion of the density of states, there are fewer fermions to which bosonic states can decay. At the Hartree level the presence of bosons and the existence of a gap in the fermionic spectrum are two sides of the same coin. These pair fluctuation approaches differ from other boson-fermion models ${ }^{15}$ of high temperature superconductors because here the bosons naturally dissociate into fermions, and similarly fermion pairs may recombine.

Hartree approaches to pair fluctuations also differ from the widely discussed phase fluctuation scenario which focuses on fluctuations in the order parameter, and underlying Mott physics. In the pair fluctuation approach the emphasis is on the observed small $\xi$. Because it is a mean field theory, this scheme is not appropriate to the critical regime and critical exponents here belong to the Gaussian rather than $3 d-X Y$ class. While Coulombic effects are profound for order parameter fluctuations in the ordered state, here they are less important, for precisely the same reasons that they are omitted in traditional TDGL ${ }^{7}$. As in BCS theory, Coulomb interactions may also enter the binding and unbinding of pairs insofar as they renormalize or even, for non- $s$ wave superconductors ${ }^{20}$, stabilize the attraction between fermions.

While there are microscopic theories ${ }^{\underline{7}}$ which serve to justify TDGL, the related diagrammatic formalism can become prohibitively complex and as a consequence, the bosonic contributions to transport properties are more readily deduced ${ }^{7}$ using TDGL theory directly. This, then, leads to the present focus on establishing a quantum extension of TDGL which is thereby amenable to detailed transport studies, at higher temperatures $T^{*}$. To proceed, we construct a simplified model of charged 
bosons subject to quantum dissipation, which has TDGL (with a non-BCS parameter set) as its special limit. Pair fluctuations involve the continuous dissociation and recombination of fermion pairs. To simulate this behavior we follow Caldeira and Leggett and treat the fermions as reservoir harmonic oscillators $\frac{18,19}{}$ coupled to the bosons. Coulomb and hard core interactions between bosons are treated at the same level as in Hartree-approximated TDGL.

We end by summarizing the key experiments we will address here. We focus on those experiments where the bosonic contributions dominate over their fermionic counterparts. It is convenient to define the electrical current $\mathbf{J}^{\mathbf{0}}=\sigma \mathbf{E}+\alpha(-\nabla T)$. In contrast to the behavior in a Fermi liquid, a sizeable Nernst signal $\nu$ (reflecting a combination of components of the $\alpha$ and $\sigma$ tensors $^{2}$ ) appears at an onset temperature appreciably above $T_{c}$, called $T_{\nu}^{*}$; this temperature is loosely associated with $T^{*}$. In the insulating phase, the thermoelectric response function $\alpha_{x y}$, as well as $\nu$ vanish at $T=0$. Precursor effects are similarly observed ${ }^{3}$ in the imaginary component, $\sigma_{2}$, of $\sigma$ at $100 \mathrm{GHz}$, which set in below $T_{\sigma}^{*}$, but appreciably above $T_{c}$. Thus far, these onset temperatures are significantly below their counterparts for $\alpha_{x y}$. Moreover, for the range of frequencies measured, these complex conductivity data can be fit ${ }^{3}$ to a rescaled Kosterlitz-Thouless form which allows extraction of a phase correlation time $\tau^{\prime}(T)$. In view of the above $\sigma$ and $\alpha$ experiments, it has seemed rather mysterious that the diamagnetic magnetization ${ }^{2}$, has a relatively non-existent normal state precursor.

Finally, the dc resistivity has been measured somewhat systematically $y^{27,28,29}$ in the underdoped regime. There is evidence for an onset temperature $T_{\rho}^{*}$ of roughly the same order of magnitude as in the other transport experiments. At this temperature an enhancement of the conductivity is evident. However, the magnitude of the deviation from the fermionic contribution is not noticeably large except in the immediate vicinity of $T_{c}$. Analysis of these data do, however, depend on assigning a particular temperature dependence to this background fermionic contribution.

\section{THE UNCORRELATED BOSON MODEL}

\section{A. Revisiting Time Dependent Ginsburg Landau Theory}

The time dependent Ginsberg-Landau equation (TDGL)

$$
\begin{aligned}
& \gamma\left(i \frac{\partial}{\partial t}-q \varphi(\mathbf{x}, t)\right) \psi(\mathbf{x}, t)=\frac{(-i \nabla-q \mathbf{A}(\mathbf{x}, t))^{2}}{2 m} \psi(\mathbf{x}, t) \\
& \quad+a_{0}(T) \psi(\mathbf{x}, t)+a^{\prime}|\psi(\mathbf{x}, t)|^{2} \psi(\mathbf{x}, t)+D(\mathbf{x}, t)
\end{aligned}
$$

is a natural starting point for characterizing the dynamics of bosons. This equation describes the fluctuational regime of conventional superconductors in the vicinity of $T_{c}$, where the bosonic degrees of freedom are represented by the wavefunction $\psi(\mathbf{x}, t)$. Here $|q|=2 e$ represents the bosonic charge, $(\varphi, \mathbf{A})$ is the electromagnetic potential which determines the EM fields via the usual formulae $\mathbf{E}(\mathbf{x}, t)=-\nabla \varphi(\mathbf{x}, t)-\frac{\partial}{\partial t} \mathbf{A}(\mathbf{x}, t)$, and $\mathbf{B}(\mathbf{x}, t)=\nabla \times \mathbf{A}(\mathbf{x}, t)$. The units adopted in this paper correspond to SI, with $\hbar=\epsilon_{0}=k_{B}=1$. The dynamics is importantly controlled by $D(\mathbf{x}, t)$ which is a white noise function satisfying

$$
\left\langle D^{*}\left(\mathbf{x}^{\prime} t^{\prime}\right) D(\mathbf{x}, t)\right\rangle=2 \gamma_{2} T \delta\left(t-t^{\prime}\right) \delta\left(\mathbf{x}-\mathbf{x}^{\prime}\right),
$$

Generally we contemplate complex $\gamma$ with $\gamma_{2} \equiv \operatorname{Im} \gamma$ and $\gamma_{1} \equiv \operatorname{Re} \gamma$.

In this paper we focus on a description in which bosonic degrees of freedom may be treated as uncorrelated. For the TDGL case this corresponds to applying a Hartree approximation to Eq. (11) so that,

$$
\begin{aligned}
& \gamma\left(i \frac{\partial}{\partial t}-q \varphi(\mathbf{x}, t)\right) \psi(\mathbf{x}, t)=\frac{(-i \nabla-q \mathbf{A}(\mathbf{x}, t))^{2}}{2 m} \psi(\mathbf{x}, t) \\
&+a(T) \psi(\mathbf{x}, t)+D(\mathbf{x}, t),
\end{aligned}
$$

Here $a(T)$ represents the (absolute value of the) bosonic chemical potential which vanishes at the superconducting transition temperature $T_{c}$, which is to be distinguished from $T_{c}^{\mathrm{mf}}$ at which $a_{0}=0$. Frequently one ignores the quartic term altogether, as in strict Gaussian fluctuation theories ${ }^{9}$. An interpretation as well as justification for TDGL can be provided by microscopic Tmatrix approaches, based on the well known AslamazovLarkin diagrams ${ }^{7}$. With the inclusion of Hartree effects ${ }^{14}$ the fluctuations have characteristic Gaussian exponents while Hartree self consistency contributions lead to a slightly modified T-matrix scheme ${ }^{10}$.

At the more macroscopic level of Eq. (1) the fermions in a superconductor are irrelevant. Nevertheless, microscopic theory makes it clear that the bosonic degrees of freedom correspond to fermion pairs; moreover, it is the fermions which are ultimately responsible for the complex noise parameter $\gamma$. The self consistent Hartree approximation introduces a fermionic exitation gap 14 (or "pseudogap") which is present at the onset of superconductivity. This depletion in the density of states is responsible for the fact that the transition temperature (contained in $a(T))$ is lowered, relative to $T_{c}^{\mathrm{mf}}$.

Despite its significant success in describing conventional superconducting fluctuations, TDGL has known limitations $\underline{16}$. In the context of understanding high $T_{c}$ superconductors, one of the most serious of these is the necessity of introducing artificial cut-offs in the fluctuational spectrum 17 , often to depress the fluctuational contributions to transport. Other extensions of TDGL have been proposed which involve introducing a modification $^{15}$ to the BCS-derived parameter set of Eq.(11), although for some experiments ${ }^{9}$ strict BCS theory appears to work quite well.

What is most perplexing about high $T_{c}$ superconductors is the appearance of "pseudogap" effects with on- 
set temperature $T^{*}$. As $T^{*}$ progressively increases away from $T_{c}$, a strict BCS approach to fluctuation-based calculations of transport appears to be invalidated ${ }^{9}$. It is the premise of the present paper that the precursor superconductivity of the pseudogap phase evolves continuously from the conventional fluctuation behavior seen, for example, in overdoped high $T_{c}$ samples. While one does not expect TDGL to hold for $T$ significantly larger than $T_{c}$, our goal here is to propose a natural extension of this theory appropriate for $T$ well above $T_{c}$. In this regime, the relaxation time of the bosons becomes comparable to or smaller than $\hbar / k_{B} T$; thus, the classical fluctuationdissipation in TDGL should be replaced by a suitable quantum counterpart. In this way we treat $\psi$ in Eq.(1) as a field theoretic operator representing the annihilation of bosons. One of the most important parameters in this extended TDGL theory is $T^{*}$ which will enter into the bosonic chemical potential. This is the temperature at which the number of bosons vanishes. One may view $T^{*}$ alternatively as the onset of the fermionic pseudogap. These two viewpoints are two sides of the same coin, since bosons disappear or dissociate when fermions are no longer bound.

\section{B. Pre-Formed Pair Model: Extended TDGL}

To gain a deeper understanding of the essence of TDGL and of its prior success away from the pseudogap (or underdoped) regime $^{9}$, we thus study a Hamiltonian describing bosons on a $d$-dimensional lattice coupled to a quantum reservoir. Our treatment of the reservoir has strong similarities to the approach of Caldeira and Leggett ${ }^{18}$. We consider

$$
\begin{gathered}
H=\sum_{\mathbf{u x}} \varepsilon_{\mathbf{u x}} \psi_{\mathbf{x}}^{\dagger}(t) \exp \left(-i q C_{\mathbf{u x}}(t)\right) \psi_{\mathbf{x}+\mathbf{u}}(t) \\
+\sum_{\mathbf{x}} q \varphi \psi^{\dagger} \psi+\sum_{i \mathbf{x}}\left\{\left(a_{i}+q \varphi\right) w_{i}^{\dagger} w_{i}+\eta_{i} \psi^{\dagger} w_{i}+\eta_{i}^{*} w_{i}^{\dagger} \psi\right\} \\
+\sum_{i \mathbf{x}}\left\{\left(b_{i}-q \varphi\right) v_{i}^{\dagger} v_{i}+\zeta_{i} \psi^{\dagger} v_{i}^{\dagger}+\zeta_{i}^{*} v_{i} \psi\right\} .
\end{gathered}
$$

Here $\psi_{\mathbf{x}}(t)$ is the boson annihilation operator at lattice site $\mathbf{x}$ and time $t$, Annihilation operators for the reservoir, $w_{i}$ and $v_{i}$ (with infinitesimal coupling constants $\eta_{i}$ and $\zeta_{i}$ ), are associated with positive and negative frequencies respectively, although the energies $a_{i}$ 's and $b_{i}$ 's are all positive. That two sets of reservoir operators are necessary will become clear later when we compare with standard TDGL.

Here $\varepsilon_{\mathbf{u x}}$ is the hopping matrix element of the bosons, to be distinguished from its electronic counterpart, and $C_{\mathbf{u x}}(t) \equiv \int_{0}^{1} \mathrm{~d} s u_{a} A_{a}(\mathbf{x}+s \mathbf{u}, t)$. Surface effects appear via the $\mathbf{x}$ dependence of $\varepsilon_{\mathbf{0 x}}$ which also contains the Hartree interaction between bosons.
The equations of motion of the system are given by

$$
\begin{aligned}
\left(i \frac{\partial}{\partial t}-q \varphi(\mathbf{x}, t)\right) \psi_{\mathbf{x}}(t) & =\sum_{\mathbf{u}} \varepsilon_{\mathbf{u x}} \exp \left(-i q C_{\mathbf{u x}}(t)\right) \psi_{\mathbf{x}+\mathbf{u}}(t) \\
& +\sum_{i} \eta_{i \mathbf{x}} w_{i \mathbf{x}}(t)+\sum_{i} \zeta_{i \mathbf{x}} v_{i \mathbf{x}}^{\dagger}(t) \\
\left(i \frac{\partial}{\partial t}-q \varphi(\mathbf{x}, t)\right) w_{i \mathbf{x}}(t) & =a_{i \mathbf{x}} w_{i \mathbf{x}}(t)+\eta_{i \mathbf{x}}^{*} \psi_{\mathbf{x}}(t) \\
\left(i \frac{\partial}{\partial t}-q \varphi(\mathbf{x}, t)\right) v_{i \mathbf{x}}^{\dagger}(t) & =-b_{i \mathbf{x}} v_{i \mathbf{x}}^{\dagger}(t)-\zeta_{i \mathbf{x}}^{*} \psi_{\mathbf{x}}(t)
\end{aligned}
$$

This model is manifestly gauge invariant.

We solve Eqs. (5b) and (5c) in the temporal gauge $(\varphi(\mathbf{x}, t) \equiv 0)$ to express $w_{i}$ and $v_{i}^{\dagger}$ in terms of both $\psi_{\mathbf{x}}(t)$ and their values at an initial time $t_{0} \rightarrow-\infty$. Substituting into Eq. (5a), we find

$$
\begin{aligned}
i \frac{\partial}{\partial t} \psi_{\mathbf{x}}(t)= & \sum_{\mathbf{u}} \varepsilon_{\mathbf{u x}} \exp \left(-i q C_{\mathbf{u x}}\right) \psi_{\mathbf{x}+\mathbf{u}}(t) \\
& -i \int_{t_{0}}^{t} \mathrm{~d} t^{\prime} \Sigma_{2}\left(t-t^{\prime}, T_{\mathbf{x}}\right) \psi_{\mathbf{x}}\left(t^{\prime}\right)+D_{\mathbf{x}}(t)
\end{aligned}
$$

where $T_{\mathbf{x}}$ is the local temperature and $\Sigma_{2}(t)$ the Fourier transform of

$$
\widetilde{\Sigma_{2}}(\omega) \equiv 2 \pi \sum_{i}\left|\eta_{i}\right|^{2} \delta\left(\omega-a_{i}\right)-2 \pi \sum_{i}\left|\zeta_{i}\right|^{2} \delta\left(\omega+b_{i}\right),
$$

It is reasonable to assume that this self energy, like any other, is smooth and that it vanishes as $\omega \rightarrow \pm \infty$. Here

$$
\begin{aligned}
D_{\mathbf{x}}(t) \equiv \sum_{i} \exp \left(-i a_{i \mathbf{x}}\left(t-t_{0}\right)\right) \eta_{i \mathbf{x}} w_{i \mathbf{x}}\left(t_{0}\right) \\
+\sum_{i} \exp \left(+i b_{i \mathbf{x}}\left(t-t_{0}\right)\right) \zeta_{i \mathbf{x}} v_{i \mathbf{x}}^{\dagger}\left(t_{0}\right)
\end{aligned}
$$

is a function which represents a generalized or quantum noise. The coupling between the $\psi$ field and each reservoir field is infinitesimal, so that the reservoir satisfies ideal Bose statistics and

$$
\begin{array}{r}
\left\langle D_{\mathbf{x}^{\prime}}\left(t^{\prime}\right)^{\dagger} D_{\mathbf{x}}(t)\right\rangle=\delta_{\mathbf{x x}^{\prime}} \int \frac{\mathrm{d} \omega}{2 \pi} \widetilde{\Sigma_{2}}\left(\omega, T_{\mathbf{x}}\right) b\left(\omega, T_{\mathbf{x}}\right) \\
\cdot \exp \left(-i \omega\left(t-t^{\prime}\right)\right)
\end{array}
$$

with $b(\omega, T) \equiv 1 /(\exp (\omega / T)-1)$ the Bose function. The physics of the bosons, transport, magnetization, specific heat, density, etc, are governed by Eqs. (6) and (9).

The reservoir parameters $a_{i}, b_{i}, \eta_{i}$ and $\zeta_{i}$ are all subsumed into the boson self energy $\widetilde{\Sigma_{2}}(\omega)$. From this point forward we ignore these quantities in favor of the boson self energy. Moreover, $\widetilde{\Sigma_{2}}(\omega)$ depends only on $\omega$ for our localized reservoir. This simplification is supported principally by the fact that this model captures the key physics found in microscopic schemes ${ }^{5.21}$, where it is sufficient to consider just the leading order $\mathbf{k}$ dependences. Differences between this previous strong coupling 
T-matrix approach ${ }^{5,21}$ and the present phenomenological boson model, are to be associated with the fact that the former scheme assumes that the T-matrix has fermionic constituents. These lead to a fine $\omega$ structure arising from the fermionic pseudogap ${ }^{21}$, as well as to different high $\omega$ asymptotics. The ensuing simplification of boson physics, however, makes our model far more tractable.

In the spatially uniform case, the Fourier transform of Eq. (6) takes a simple form:

$$
\left(\omega-\varepsilon(\mathbf{k})-\widetilde{\Sigma_{1}}(\omega)+\frac{i}{2} \widetilde{\Sigma_{2}}(\omega)\right) \widetilde{\psi}(\mathbf{k}, \omega)=\widetilde{D}(\mathbf{k}, \omega),
$$

with $\varepsilon(\mathbf{k}) \equiv \sum_{\mathbf{u}} \varepsilon_{\mathbf{u}} \exp (i \mathbf{k u})$, and

$$
\widetilde{\Sigma_{1}}(\omega) \equiv P \int \frac{\mathrm{d} \omega^{\prime}}{2 \pi} \frac{1}{\omega-\omega^{\prime}} \widetilde{\Sigma_{2}}\left(\omega^{\prime}\right)
$$

We are now in a position to clarify the relationship between this theory and TDGL. When $T$ is close to $T_{c}$, the bosonic relaxation rate $\tau^{-1}$ is considerably smaller than $T$ (typically of the order of tens or hundreds of Kelvin). In this regime the dynamics is dominated by low frequencies $|\omega| \sim \tau^{-1} \ll T$, and the Bose function is well approximated by $b(\omega, T) \approx T / \omega$. We presume that the high energy cut-off scale $\Omega$ in $\widetilde{\Sigma_{2}}(\omega)$, which is associated with the reservoir, is of the order of typical electronic energies, thus thousands of $\mathrm{K}$. Then, at low frequencies, the bosonic self energy is given by the linear functions

$$
\widetilde{\Sigma_{1}}(\omega)-\widetilde{\Sigma_{1}}(0) \approx\left(1-\gamma_{1}\right) \omega
$$

and

$$
\widetilde{\Sigma_{2}}(\omega) \approx 2 \gamma_{2} \omega
$$

where from Eq. (7), $\widetilde{\Sigma_{2}}(\omega)$ vanishes at zero frequency.

With the above approximations the quantum noise correlation function Eq. (9) is reduced to its classical limit, given by Eq. (2). In the same way Eq. (10) is reduced to Eq. (3), with the important parameter

$$
-\mu_{\text {pair }} \equiv a(T)=\min _{\mathbf{k}} \varepsilon(\mathbf{k})+\widetilde{\Sigma_{1}}(0) .
$$

We may now see that the two sets of reservoir fields, $w_{i}$ and $v_{i}$, are associated with opposite charges, $q$ and $-q$; they contribute to the positive and negative frequency regimes of $\widetilde{\Sigma_{2}}(\omega)$, respectively. If either of these two were omitted, $\widetilde{\Sigma_{2}}(\omega)$ would vanish on one side of the origin; thus, the slope of $\widetilde{\Sigma_{2}}(\omega)$ would be discontinuous across zero frequency, and one would never arrive at the TDGL limit, no matter how small the frequency. In summary, the boson model presented in this paper is a natural quantum extension of TDGL. Conversely, TDGL is the low frequency limit of our quantum boson model.

In preparation for computing transport coefficients, we introduce the bosonic correlation function, which can be derived from Eqs. (10) and (9). This is given by

$$
\left\langle\widetilde{\psi}^{\dagger}\left(\mathbf{k}^{\prime} \omega^{\prime}\right) \widetilde{\psi}(\mathbf{k}, \omega)\right\rangle=\widetilde{A}(\mathbf{k}, \omega) b(\omega)(2 \pi)^{d+1} \delta\left(\mathbf{k}-\mathbf{k}^{\prime}\right) \delta\left(\omega-\omega^{\prime}\right)
$$

where $\widetilde{A}(\mathbf{k}, \omega)=\operatorname{Re} 2 i \widetilde{\mathcal{T}}(\mathbf{k}, \omega)$ is the boson spectral function, and

$$
\widetilde{\mathcal{T}}(\mathbf{k}, \omega) \equiv\left(\omega-\varepsilon(\mathbf{k})-\widetilde{\Sigma_{1}}(\omega)+\frac{i}{2} \widetilde{\Sigma_{2}}(\omega)\right)^{-1}
$$

is the boson propagator or "T-matrix". The average number of bosons per lattice site is

$$
n \equiv\left\langle\psi^{\dagger} \psi\right\rangle=v \int \frac{\mathrm{d}^{d} k}{(2 \pi)^{d}} \frac{\mathrm{d} \omega}{2 \pi} \widetilde{A}(\mathbf{k}, \omega) b(\omega),
$$

where $v$ is the cell volume associated with each lattice site.

\section{Transport Coefficients}

The electric current $\mathbf{J}^{0}$ and heat current $\mathbf{J}^{1}$ follow from charge and energy conservation:

$$
\begin{aligned}
& J_{a}^{n}(\mathbf{x}, t)=\frac{q^{1-n}}{2 v}\left\{\left(i \frac{\partial}{\partial t}-q \varphi(\mathbf{x}, t)\right)^{n} \psi(\mathbf{x}, t)\right\}^{\dagger} \\
& \cdot \sum_{\mathbf{u}} i u^{a} \varepsilon_{\mathbf{u x}} \exp \left(-i q C_{\mathbf{u x}}(t)\right) \psi_{\mathbf{x}+\mathbf{u}}(t)+\text { h.c. }
\end{aligned}
$$

When a magnetic field $B_{a b} \equiv \frac{\partial}{\partial x_{a}} A_{b}-\frac{\partial}{\partial x_{b}} A_{a}$ is applied to the system, surface electric and heat currents appear in a thin shell around its boundary.

To derive these surface magnetizations we proceed as follows. We confine bosons within the sample boundaries via a spatially dependent $\varepsilon_{\mathbf{0 x}}$ which approaches the bulk value well away from the surface and $+\infty$ on the boundary. We presume that the spatial gradient of $\varepsilon_{\mathbf{0} \mathbf{x}}$ is small, as is the applied magnetic field so that we may calculate the electric and heat currents to leading order in these quantities. Integrating the current in the normal direction of the surface, from the boundary to deep within the sample, we obtain

$$
M_{a b}^{n}=\frac{q^{2-n}}{6} B_{c d} \int \frac{\mathrm{d}^{d} k}{(2 \pi)^{d}} \frac{\mathrm{d} \omega}{2 \pi} \Delta_{a b c d} \operatorname{Re} i \widetilde{\mathcal{T}}(\mathbf{k}, \omega)^{2} \omega^{n} b(\omega),
$$

where $M_{a b}^{0}$ is the usual magnetization, $M_{a b}^{1}$ is the thermal analogue ${ }^{22}$ of $M_{a b}^{0}, \quad \Delta_{a b c d}(\mathbf{k}) \equiv \frac{1}{2}\left(v_{a c}(\mathbf{k}) v_{b d}(\mathbf{k})\right.$ $\left.-v_{a d}(\mathbf{k}) v_{b c}(\mathbf{k})\right)$, and $v_{a b}(\mathbf{k}) \equiv \frac{\partial^{2}}{\partial k_{a} \partial k_{b}} \varepsilon(\mathbf{k})$ is the inverse mass tensor of the bosons, whose group velocity is given by $v_{a}(\mathbf{k}) \equiv \frac{\partial}{\partial k_{a}} \varepsilon(\mathbf{k})$.

When an external magnetic field is present, the bulk volume current $\mathbf{J}^{n}$ must be combined with surface contributions so that the net "transport" currents are given by ${ }^{9}$ :

$$
J_{(\operatorname{tr}) a}^{n}=\left\langle J_{a}^{n}\right\rangle+\frac{\partial M_{a b}^{n}}{\partial T} E_{b}^{1}+\delta_{n, 1} M_{a b}^{0} E_{b}^{0}
$$

For small, but constant $B_{a b}$, electric field $E_{a}^{0} \equiv$ $E_{a}$, and negative thermal gradient $E_{a}^{1} \equiv-\frac{\partial}{\partial x_{a}} T$, we obtain the linearized response functions $J_{(\operatorname{tr}) a}^{n}=$ 
$\sum_{n^{\prime}=0}^{1} \sum_{b} L_{a b}^{n n^{\prime}} E_{b}^{n^{\prime}}$, and the DC transport coefficients $L_{a b}^{n n^{\prime}}$ can be compactly written as

$$
\begin{aligned}
& L_{a b}^{n n^{\prime}}=\frac{q^{2-n-n^{\prime}}}{2 T^{n^{\prime}}} \int \frac{\mathrm{d}^{d} k}{(2 \pi)^{d}} \frac{\mathrm{d} \omega}{2 \pi} v_{a} v_{b} \widetilde{A}^{2} \omega^{n+n^{\prime}} b^{(1)} \\
& +\frac{q^{3-n-n^{\prime}} B_{c d}}{6 T^{n^{\prime}}} \int \frac{\mathrm{d}^{d} k}{(2 \pi)^{d}} \frac{\mathrm{d} \omega}{2 \pi} v_{a} v_{c} v_{b d} \widetilde{A}^{3} \omega^{n+n^{\prime}} b^{(1)}
\end{aligned}
$$

where $b^{(1)}(\omega) \equiv-\frac{\partial b(\omega)}{\partial \omega}$ arises from the $\omega=0$ limit, in which the boson absorbs or emits an infinitesimal amount of energy in the process of making a transition to an adjacent energy level. $L_{a b}^{00}=\sigma_{a b}$ is the isothermal electric conductivity, $L_{a b}^{11}$ is the isoelectropotential thermal conductivity, and $L_{a b}^{01}=\alpha_{a b}$ and $L_{a b}^{10}$ are off-diagonal coefficients. Eq. (21) satisfies the Onsager relation ${ }^{9} T^{n^{\prime}} L_{a b}^{n n^{\prime}}(\overleftrightarrow{B})=T^{n} L_{b a}^{n^{\prime} n}(-\overleftrightarrow{B})$ (no summation), as a consequence of our inclusion of the surface terms 9 . Surface effects enter into $L_{a b}^{01}, L_{a b}^{10}$ and $L_{a b}^{11}$, but cancel in $L_{a b}^{00}$.

Finally, we deduce the boson contribution to the complex ac conductivity and ac Hall conductivity

$$
\begin{gathered}
\widetilde{\sigma}_{a b}(\omega)=\frac{i q^{2}}{\omega} \int \frac{\mathrm{d}^{d} k^{\prime}}{(2 \pi)^{d}} \frac{\mathrm{d} \omega^{\prime}}{2 \pi} v_{a}\left(\mathbf{k}^{\prime}\right) v_{b}\left(\mathbf{k}^{\prime}\right) \widetilde{A}\left(\mathbf{k}^{\prime} \omega^{\prime}\right) b\left(\omega^{\prime}\right) \\
\cdot\left(\widetilde{\mathcal{T}}\left(\mathbf{k}^{\prime}, \omega^{\prime}+\omega\right)+\widetilde{\mathcal{T}}^{*}\left(\mathbf{k}^{\prime}, \omega^{\prime}-\omega\right)-\widetilde{\mathcal{T}}\left(\mathbf{k}^{\prime} \omega^{\prime}\right)-\widetilde{\mathcal{T}}^{*}\left(\mathbf{k}^{\prime} \omega^{\prime}\right)\right) \\
+\frac{i q^{3}}{2 \omega} B_{c d} \int \frac{\mathrm{d}^{d} k^{\prime}}{(2 \pi)^{d}} \frac{\mathrm{d} \omega^{\prime}}{2 \pi} v_{a}\left(\mathbf{k}^{\prime}\right) v_{c}\left(\mathbf{k}^{\prime}\right) v_{b d}\left(\mathbf{k}^{\prime}\right) \widetilde{A}\left(\mathbf{k}^{\prime} \omega^{\prime}\right) b\left(\omega^{\prime}\right) \\
\cdot\left(i \widetilde{\mathcal{T}}\left(\mathbf{k}^{\prime}, \omega^{\prime}+\omega\right)-i \widetilde{\mathcal{T}}^{*}\left(\mathbf{k}^{\prime}, \omega^{\prime}-\omega\right)\right) \\
\cdot\left(\widetilde{\mathcal{T}}\left(\mathbf{k}^{\prime}, \omega^{\prime}+\omega\right)+\widetilde{\mathcal{T}}^{*}\left(\mathbf{k}^{\prime}, \omega^{\prime}-\omega\right)-\widetilde{\mathcal{T}}\left(\mathbf{k}^{\prime} \omega^{\prime}\right)-\widetilde{\mathcal{T}}^{*}\left(\mathbf{k}^{\prime} \omega^{\prime}\right)\right) .
\end{gathered}
$$

It can be verified that $\widetilde{\sigma}_{a b}(\omega \rightarrow 0)=L_{a b}^{00}$ and that Eq. (22) satisfies the f-sum rule.

\section{PHENOMENOLOGY}

In this section we arrive at a simple and generic phenomenology describing the pre-formed pairs or bosons of our extended TDGL model, in the context of holedoped cuprates. Our goal in the next section is to use this phenomenology to address transport data at a semiquantitative level with as few fitting parameters as possible. It should be noted that this phenomenology is generally compatible with previously derived T-matrix based approaches $\underline{\underline{6}}$. The most important component of this phenomenological discussion lies in our introduction of the parameter $T^{*}$, which has no natural counterpart in TDGL approaches. This is the temperature at which the number of bosons vanishes. This same temperature is reflected in the fermionic spectrum as that at which the excitation (pseudo)gap vanishes, so that fermions are no longer bound into bosons. The remaining parameter choices discussed below are reasonably straightforward, and represent natural extensions based on TDGL theory.
There are three factors which govern the dynamics of our bosons: the dispersion function $\varepsilon(\mathbf{k})$, the self energy $\widetilde{\Sigma}(\omega)$, and the chemical potential $\mu_{\text {pair }}$. We begin with $\varepsilon(\mathbf{k})$, which is given by $\mathcal{E}_{a}\left(1-\cos \left(k_{a} s_{a}\right)\right)+$ $\mathcal{E}_{b}\left(1-\cos \left(k_{b} s_{b}\right)\right)+\mathcal{E}_{c}\left(1-\cos \left(k_{c} s_{c}\right)\right)+$ const., presuming only nearest neighbor hopping. Here $s_{c} \equiv s$ is the interlayer spacing, while $s_{a}$ and $s_{b}$ are in-plane lattice constants. The characteristic boson bandwidths $\left(\mathcal{E}_{a, b}\right)$ satisfy $\mathcal{E}_{a} \approx \mathcal{E}_{b}$ of the order of a few thousand $\mathrm{K}^{32}$. By contrast, $\mathcal{E}_{c}$ is on the order $10 \mathrm{~K}$ for the least anisotropic cuprates (e.g., YBCO) and roughly two orders of magnitude smaller for the most anisotropic systems (e.g, $\mathrm{Bi2212})$. In this way the dispersion function may be further simplified to yield $k_{a b}^{2} / 2 m_{a}+\mathcal{E}_{c}\left(1-\cos \left(k_{c} s\right)\right)+$ const., the well known Lawrence Doniach dispersion.

Next we turn to $\widetilde{\Sigma}(\omega)$, which is expected to be given by its TDGL form. Eqs. (12) and (13), for frequencies much lower than a characteristic cutoff energy $\Omega$, introduced earlier. Moreover, in the fermionic regime, far from the true bosonic limit, (as appears to be the case for the cuprates), $\gamma_{1} \ll \gamma_{2}$. Our results are, thus, rather insensitive to $\gamma_{1}$ and for simplicity we set it to zero $\underline{\underline{30}}$

To obtain an estimate of $\Omega$, we use our model to calculate the average number of bosons per lattice site $n(T)$. At $T_{c}$ we obtain the simple result 33 :

$$
n\left(T_{c}\right) \approx \frac{\widetilde{\Omega}}{4 \pi \widetilde{\mathcal{E}}_{a}}+\frac{T_{c}}{2 \pi \mathcal{E}_{a}} \ln \frac{2 T_{c}}{\widetilde{\mathcal{E}}_{c}},
$$

where, for general energy scales $E$ we define $\widetilde{E} \equiv E / \gamma_{2}$. It is reasonable to assume that well into the underdoped regime $n\left(T_{c}\right)$ falls somewhere inside the range 0.01-0.5 electron pairs per lattice site. Since $T_{c}$ is considerably smaller than $\widetilde{\mathcal{E}}_{a}$, the first term on the right side of Eq. (23) dominates, and we deduce that $\Omega$ is of the same order of magnitude as $\mathcal{E}_{a}$, thus thousands of K. The above analysis indicates that the simple linearized expansion of the boson self energy, given by Eqs. (12) and (13), is a reasonably good approximation over the range of relevant frequencies we consider here.

Finally we address the quantity $\mu_{\text {pair }}(T)$ which depends on the important temperature $T^{*}$ which has no natural counterpart in TDGL theory. This is the temperature where $\mu_{\text {pair }}$ diverges. At this temperature the Bose degrees of freedom vanish. Concomitantly, at $T^{*}$ the fermionic excitation gap disappears, although, presumably this is a crossover scale rather than a sharp transition. It has been argued that TDGL approaches overestimate fluctuation effects so that short wavelength cut-offs in the fluctuational spectrum have to be introduced, most recently to address the paraconductivity 17 . In the boson model introduced here, the non-TDGL temperature dependence in the pair chemical potential, $\mu_{\text {pair }}$, removes the necessity for introducing an ad hoc cut-off.

We may estimate the magnitude of $\mu_{\text {pair }}$ at intermediate temperatures between $T_{c}$ and $T^{*}$, by using the boson density $n(T)$. T-matrix based theories suggest $\underline{\underline{6}}$, that the electronic pseudogap scales with the number of bosons 
above $T_{c}$. A key assumption of our approach is that the magnitude of the pseudogap decreases by an appreciable fraction at intermediate temperatures between $T_{c}$ and $T^{*}$. This assumption is reasonably compatible with experiments such as ARPES, but it has not been conclusively established at this time. We expect that the number of bosons evolves in a similar fashion.

Within our theoretical model, we require that $-\mu_{\text {pair }} \sim$ $\Omega$ in order to supress $n(T)$ significantly relative to $n\left(T_{c}\right)$, for $T$ between $T_{c}$ and $T^{* 34}$. TDGL expressions for $\mu_{\text {pair }}$ $\left(\mu_{\text {pair }} \propto\left(T-T_{c}\right)\right)$ will not lead to a sufficiently rapid decline in the boson number. It follows that there must be an additional term in the pair chemical potential which is negligible in the vicinity of $T_{c}$, but which rapidly increases at higher temperatures, and diverges at $T^{*}$. A form generally compatible with the above analysis, as well as with microscopic T-matrix based schemes ${ }^{6}$, can be written as

$$
\tau^{-1}(T) \equiv-\frac{\mu_{\mathrm{pair}}}{\gamma_{2}}=\frac{8}{\pi \eta}\left(T-T_{c}\right)+c \frac{\left(T-T_{c}\right)^{3}}{\left(T^{*}-T\right)^{2}},
$$

where we have introduced a quantity $\eta$ which represents the ratio of the boson coherence time $\tau$ to its BCS value, $\pi /\left(8\left(T-T_{c}\right)\right)$, for $T \sim T_{c}$. Throughout, we take $c \sim$ 15 , consistent with our order of magnitude estimates of $\mu_{\text {pair }}$. It should be stressed that there is nothing in this and the following sections which depends on the specific details of this functional form, provided the non-TDGL contribution (or second term) diverges at $T^{*}$ and vanishes sufficiently rapidly as $T$ approaches $T_{c}$.

Both ac and dc conductivity data indicate that $\eta$ is close to 1 for optimally doped cuprates ${ }^{26}$ but it becomes significantly larger than 1 as the material is progressively underdoped ${ }^{3}$. In this way the pairs live longer in the vicinity of $T_{c}$ than expected from BCS theory. Quantitative analysis of the ac conductivity data (presented in the next section) shows that $\eta$ can be of the order 10-20 for a typical underdoped Bi2212 sample. We take $\eta$ as the only essential fitted parameter in all of our numerical analysis ${ }^{31}$. Other parameter choices ${ }^{35}$ throughout this paper are for illustrative purposes only.

We end this section by noting that there is an additional quantum statistical effect which acts to further suppress boson transport. This is associated with the fact that the factor $-b^{\prime}(\omega)$ is smaller than its TDGL counterpart $T / \omega^{2}$. This effect is appreciable when $\tau \sim 1 / T$, and greatly amplified when $\tau \ll 1 / T$, thereby further suppressing bosonic contributions to (DC) transport.

\section{NUMERICS AND QUALITATIVE COMPARISON WITH EXPERIMENT}

\section{A. Nernst Effect and Magnetization}

In Fig. 1 we plot the transverse thermoelectric coefficient $\alpha_{x y}$ vs $T$ for three underdoped samples with indicated $T^{*} / T_{c}$. The arrows show the corresponding values of $T_{c}$ which progressively decrease as $T^{*} / T_{c}$ increases. The inset plots (unpublished) experimental data on $\mathrm{La}_{2-x} \mathrm{Sr}_{x} \mathrm{CuO}_{4}$ from Ref. 23 which curves are for $x=0.12,0.07$, and 0.03 and which are in rough correspondence to the values of $T^{*} / T_{c}$ in the three theory plots. The onset temperatures are of the order of $T^{*} / 2$, as in experiment. In the vicinity of $T_{c}$, the calculated behavior of $\alpha_{x y}$ corresponds to that of TDGL theory, albeit with modified coefficients. Thus $\alpha_{x y}$ diverges at $T_{c}$ although $\nu$ is finite there ${ }^{9}$. Similarly, with overdoping the behavior becomes characteristic of a more conventional fluctuation picture ${ }^{9}$ in which $T^{*} \approx T_{c}$. The essential distinction between TDGL and the present case is that the onset temperature for bosonic contributions can be substantially higher than the conventional fluctuation regime. Semi-quantitative agreement between theory and experiment appears quite satisfactory.

In both theory and experiment the dotted lines are for a non-superconductor with $T_{c}=0$. To apply the present theory to the insulating case we presume that the first term on the right hand side of Eq. (24) is a non-vanishing number which corresponds to the chemical potential of non-condensed electron pairs. This constant is chosen to be $24 \mathrm{~K}$ in order to fit the maximum of $\alpha_{x y}(T)$ to its experimental counterpart. This figure illustrates the fact that $\alpha_{x y}$ (as well as $\nu$ ) vanishes at $T=0$ in nonsuperconducting samples. This derives from the behavior of the Bose function which approaches a step function of $\omega$ at very low temperatures.

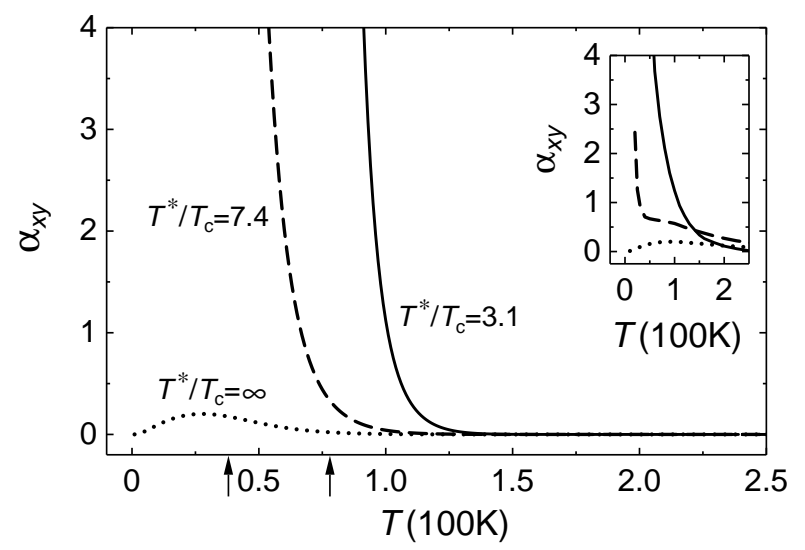

FIG. 1: Theoretical curves for the normal state $\alpha_{x y}$ with variable $T^{*} / T_{c}$; experimental counterparts ${ }^{23}$ in inset for $\mathrm{La}_{2-x} \mathrm{Sr}_{x} \mathrm{CuO}_{4}$ at $x=0.03,0.07$, and 0.12 . Both the theoretical and experimental values of $\alpha_{x y}$ are normalized by $0.02(B / \mathrm{T}) \mathrm{V} /(\mathrm{K} \Omega \mathrm{m})$, a number obtained earlier ${ }^{9}$ at $2 T_{c}$ for an optimally doped sample.

An interesting inference from the data ${ }^{2}$ is that, while, there is a considerable precursor effect for $\alpha_{x y}$, the (orbital) magnetization drops to its superconducting value only in the immediate vicinity of Bose condensation. This quantity [given as $M_{a b}^{0}$ in Eq. [19]) is plotted in the inset to Fig. 2 for the same parameter set as in Fig. 1. The fermionic background (measured experimen- 
tally) is neglible, so that the bosonic contribution necessarily dominates. The sharpness of the Meissner onset (which clearly reflects $T_{c}$ and not $T^{*}$ ) can be attributed to the small ratio of the boson velocity to the speed of light (and the small size of the hyperfine constant). Similar results hold in TDGL-based calculations.

\section{B. AC Conductivity}

In the main body of Fig. 2 are plotted the real and imaginary components of the ac conductivity as a function of $T$ for $\omega /(2 \pi) \approx 100 \mathrm{GHz}$ for two different values of $T^{*} / T_{c}$, as in the previous figure. Both $\sigma_{1}=\operatorname{Re} \sigma$ and $\sigma_{2}=\operatorname{Im} \sigma$ are finite at $T_{c}$ with $\sigma_{2}$ larger than $\sigma_{1}$. Because of the relatively "high" frequencies $\left(\omega \approx 5 \mathrm{~K} \gg \widetilde{\mathcal{E}}_{c}\right)^{35}$, the associated frequency dependence is not the asymptotically low $\omega$ limit, near $T_{c}$, where it would vary as $1 / \sqrt{\omega}$. It follows that, just as in TDGL theory, $\mathrm{d} \sigma_{1} / \mathrm{d} T$ is finite while $\mathrm{d} \sigma_{2} / \mathrm{d} T$ diverges at $T_{c}$. We find that the magnitude of $\sigma$ is about twice the experimental value at $T_{c}{ }_{c}^{3}$. This prediction is the most notable difference between theory and experiment ${ }^{3}$.

The theoretically deduced onset temperature $T_{\sigma}^{*}$, for precursor effects in $\sigma_{2}$ can be estimated by noting that the fermionic background is relatively negligible so that $\sigma_{2}$ becomes appreciable when it reaches a few percent of its value at $T_{c}$. This corresponds to onset temperatures which are factors of 2 or so closer to $T_{c}$ than those estimated from $\alpha_{x y}$.

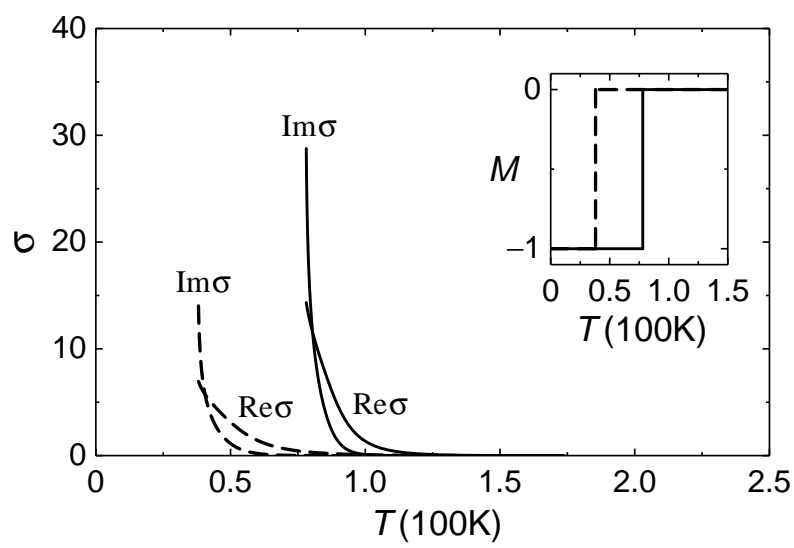

FIG. 2: Real and imaginary components of normal state $\sigma$ (normalized by $\sigma_{Q} \equiv e^{2} / s$ ) at $100 \mathrm{GHz}$ vs $T$. Solid and dashed curves have same $T^{*} / T_{c}$ as for counterparts in Fig. 1] Associated magnetizations are plotted in inset, with the external magnetic field chosen as the unit.

In Fig. 3] we replot the calculated ac conductivity (for $\left.T^{*} / T_{c}=3.1\right)$, following the Kosterlitz-Thouless (KT) based analysis of Ref 3 . In the main figure we illustrate these KT fits. These are the basis of an interpretation of conductivity data in terms of vortices above $T_{c}$. We present plots of $\phi \equiv \tan ^{-1}\left(\sigma_{2} / \sigma_{1}\right)$ and $|S|$ as a function of $\omega / \Omega^{\prime}$ obtained from $\sigma(\omega) / \sigma_{Q}=T_{\theta}^{0}(T) S\left(\omega / \Omega^{\prime}\right) \Omega^{\prime-1}$.
As in the data, here, $T_{\theta}^{0}$ and $\Omega^{\prime}$ are deduced parameters. The inset plots $\tau^{\prime}=1 / \Omega^{\prime}$ as a function of temperature. We find that $T_{\theta}^{0}$ is roughly constant in $T$, in contrast to the data which finds this quantity to be decreasing as $T$ increases. Nevertheless, the agreement with experiment for all three quantities plotted in Fig. 3 is quite good. In this way one might arque that key features of the ac conductivity data in Ref. 3 and attributed to KT physics, may equally well be explained by pre-formed pairs.

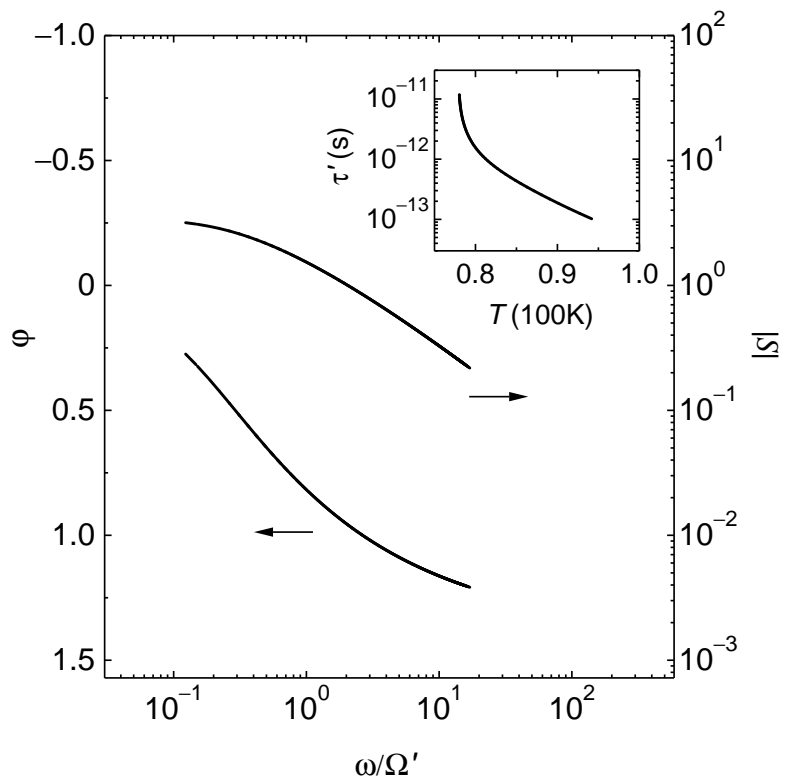

FIG. 3: Fits to Kosterlitz-Thouless (KT) scaling of the conductivity which can be compared to the analysis in Ref 3 . See text for details.

\section{Resistivity}

In the main body of Fig. 4 we plot DC resistivity curves, $\rho$ vs. $T$ for an optimal and underdoped system. In addition to the bosonic contribution we have added a fairly generic ${ }^{28}$ linear-in- $T$ contribution from the fermions, as the background term. The underdoped sample has the same parameters as in previous figures with $T^{*} / T_{c}=3.1$. For the optimally doped case we take $T^{*} / T_{c} \approx 1.45$ and $\eta \approx 1$. It is clear that the transition region is wider for the underdoped system. The increased separation between $T^{*}$ and $T_{c}$, and the much elongated boson relaxation time, are responsible for this widening. Our optimal results compare favorably with those in the literature ${ }^{26}$. For the underdoped case, the wider than experimentally observed ${ }^{27}$ transition regime that we find in $\rho$ is closely connected to the behavior seen in the ac conductivity of Figure 2. And the latter is compatible with the experiments of Ref 3 .

Dimensionality also plays an important role in determining the behavior of the paraconductivity. In the upper left inset of Fig. 4 we plot the resistivity for a compa- 


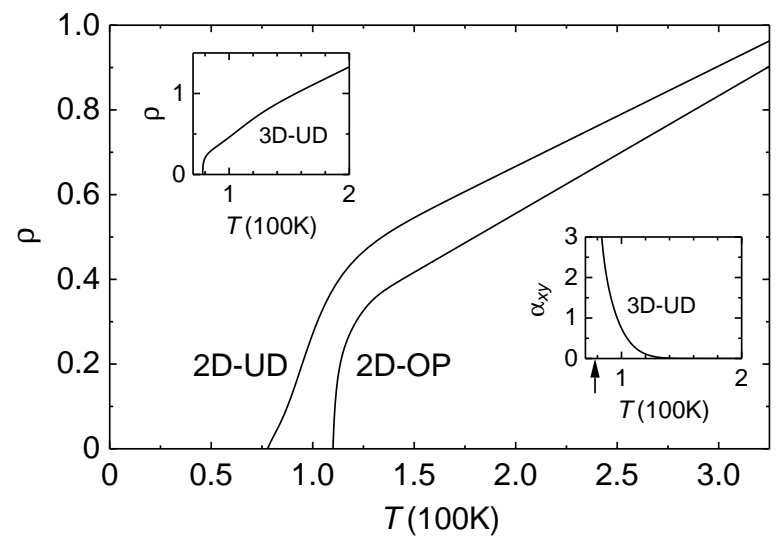

FIG. 4: DC resistivity $\rho$ (normalized by $\rho_{Q} \equiv s / e^{2}$ ) vs. $T$, for underdoped and optimally doped samples. The underdoped sample has the same parameters as in previous figures. The optimally doped sample corresponds to $\eta=1$. In the insets are plotted the $\rho$ and $\alpha_{x y}$ curves for an underdoped, more 3dimensional, system like YBCO, with $\eta=7.5$, and $T^{*} / T_{c}=$ 3.1. The arrow in the lower right inset indicates $T_{c}$.

rably underdoped, but more three dimensional material, such as YBCO. Increased 3-dimensionality (via increased c-axis coherence length) clearly shrinks the appararent transition range of $\rho$. This can be traced to the reduced bosonic density of states in the low energy regime. To see how the change of dimensionality affects the Nernst coefficient, we present a plot of $\alpha_{x y}$ in the lower inset for this same sample. The onset for Nernst is roughly $40 \mathrm{~K}$ above $T_{c}$. This contrasts sharply with that of $\rho$ which is at most a few $\mathrm{K}$ above $T_{c}$. Thus, the same sample, with exactly the same parameters, appears to have very different onset temperatures for resistivity and Nernst. It should be noted that if one looks closely at the plot of $\rho$ a small downward curvature develops well above $T_{c}$, which can be traced to $T^{*}$ effects.

\section{CONCLUSIONS}

In this paper we have investigated the effects of preformed pairs or bosonic degrees of freedom on normal state transport properties. Our goal was to provide insight into transport anomalies in the high temperature superconductors. These anomalies are associated with non-Fermi liquid-like observations, many of which seem to be rather similar to what is found in the presence of superconducting fluctuations. By contrast to the standard fluctuations of TDGL theory, however, these preformed pairs appear at relatively high temperatures $T^{*}$ compared to $T_{c}$. Throughout our discussion, it should be stressed that we view $T^{*}$ as a "crossover" temperature, rather than a sharp phase transition. The origin of these preformed pairs is not specified. They may arise from the strong attractive interaction which drives the superconductivity. (Indeed, attractive interactions in a non $s$-wave channel ${ }^{20}$ are possible, despite the presence of strong Coulombic effects). However, one may entertain as well other scenarios for the mechanism of pair formation.

In the process of addressing transport we have devised a new formalism for extending TDGL into the quantum regime, well away from the transition temperature where the bosons condense. Our approach, moreover, allows exact calculations of all transport properties and, in the immediate vicinity of $T_{c}$, our results are equivalent to those of standard TDGL. Comparison with experiment is quite satisfactory. It should be stressed that no particular fitting to the data was done, but rather in this paper we have explored the generic features of the model. Moreover, we have addressed a wide variety of different experiments: Nernst, ac and dc conductivity and diamagnetic measurements. Aside from introducing a quantum statistical treatment of the bosons, a key feature of our approach is the introduction of the important temperature $T^{*}$. This is a new concept, not present in TDGL. Pairs now form far from the critical regime. But at the same time $T^{*}$ provides an ultimate cut-off. There are no bosons (or fermionic pseudogap) beyond this regime.

Our paper shows that transport anomalies are compatible with the presence of bosonic degrees of freedom. The same inference is made from thermodynamic and ARPES experiments which deduce the onset of a gap in the fermionic spectrum. Indeed, we view these broad classes of phenomena as two sides of the same coin. One of the key conclusions of this paper is that onset temperatures for transport anomalies vary from one experiment to another. Moreover, all are considerably lower than $T^{*}$. The boson contribution to transport depends sensitively on the pair life time $\tau$. Only sufficiently long lived bosons significantly contribute to transport. Hence bosonic contributions to transport are suppressed well before $T^{*}$ is reached.

Ours should be viewed as an alternative to the vortex scenario or related phase fluctuation picture for addressing normal state transport anomalies. Indeed, it has been recently suggested ${ }^{25}$ that phase fluctuations alone cannot explain pseudogap phenomena. The reasonable agreement between the generic results of the present theory and experiment for all the figures provides support for a preformed-pair alternative to this vortex scenario. Non-condensed bosons are also present below $T_{c}$ and, thereby, will lead to some continuity between transport coefficients across the transition. In extending this work to the ordered phase, however, it will be useful to find a relationship between bosons and vortices. Along these lines is the dual representation explored earlier ${ }^{24}$ in which vortices are the basic particles, rather than Cooper pairs.

Work supported by NSF-MRSEC Grant No.DMR0213745. We thank Y. Wang and N. P. Ong for unpublished data, and I. Ussishkin and G. Mazenko for very useful conversations. 
1 Z. Xu, N. Ong, Y. Want, T. Kakeshita and S. Uchida, Nature 406, 486 (2000).

2 Y. Wang, Z. A. Xu, T. Kakeshita, S. Uchida, S. Ono, Y. Ando and N. P. Ong, Phys. Rev. B 64, 224519 (2001).

3 J. Corson, R. Mallozzi, J. Orenstein, J. N. Eckstein and I. Bozovic, Nature 398, 221 (1999).

4 V. J. Emery and S. A. Kivelson, Nature 374, 434 (1995).

5 M. Randeria, in Bose Einstein Condensation, edited by A. Griffin, D. Snoke and S. Stringari (Cambridge Univ. Press, Cambridge, 1995), pp. 355-92.

${ }^{6}$ Q. J. Chen, I. Kosztin, B. Janko and K. Levin, Phys. Rev. Lett. 81, 4708 (1998).

7 A. Larkin and A. Varlamov (2001), cond-mat/0109177.

8 S. Ullah and A. T. Dorsey, Phys. Rev. B 44, 262 (1991).

${ }^{9}$ I. Ussishkin, S. Sondhi and D. Huse, Phys. Rev. Lett. 89, 287001 (2002).

10 B. R. Patton, Phys. Rev. Lett. 27, 1273 (1971).

11 A. J. Leggett, in Modern Trends in the Theory of Condensed Matter (Springer-Verlag, Berlin, 1980), pp. 13-27.

12 P. Nozières and S. Schmitt-Rink, J. Low Temp. Phys. 59, 195 (1985).

13 J. Stajic, A. Iyengar, Q. Chen and K. Levin, Phys. Rev. B 68, 174517 (2003).

14 R.F. Hassing and J.W. Wilkins, Phys. Rev. B 7, 1890 (1973).

15 V.B. Geshkenbein, L.B. Ioffe and A.I. Larkin, Phys. Rev. B 55, 3173 (1997).

16 The white-noise leads to some divergent results, including $\left\langle\psi^{*} \psi\right\rangle$ and some transport coefficients such as the thermal conductivity.

17 E. Silva, S. Sarti, R. Fastampa and M. Giura, Phys. Rev. B 64, 144508 (2001).

18 A. Caldeira and A.J. Leggett, Physica 121A, 587 (1983).

19 Francisco Guinea, Phys. Rev. Lett. 53, 1268 (1984).

20 D.Z. Liu and K. Levin, Physica C 275, 81-86 (1997).

21 J. Maly, B. Janko and K. Levin, Physica C 321, 113 (1999).

22 In the present case there is no particle-hole symmetry and we consider $T$ away from $T_{c}$ so that the thermal surface magnetization is non-zero.
23 Yayu Wang and N. P. Ong, private communication.

24 M.A. Fisher and D.H. Lee, Phys. Rev. B 39, 2756 (1989).

${ }^{25}$ P.A. Lee (2003), cond-mat/0307508.

26 G. Balestrino et al, Phys. Rev. B 46, 14919 (1992).

27 T. Watanabe, T. Fujii and A. Matsuda, Phys. Rev. Lett. 79, 2113 (1997).

28 B. Leridon et al, Phys. Rev. Lett. 87, 197007 (2001).

29 S.R. Currás et al, Phys. Rev. B 68, 094501 (2003).

30 If we are to study the Hall conductivity etc, the first order term in $\gamma_{1}$ is important, since the zeroth order term vanishes. See Ref 9 .

31 For the underdoped samples, illustrated in Figs. 12 and 3 and the underdoped curve in the main body of Fig. 4 we choose $\eta=20$ to fit the onset temperature of $\operatorname{Im} \sigma$ at $100 \mathrm{GHz}^{3}$.

32 Here the quantity that is determined is actually $\mathcal{E}_{a} / \gamma_{2}$. A similar situation holds for $\mathcal{E}_{c}$ and $\mu_{\text {pair }}$. Due to the approximate linearity of $\widetilde{\Sigma}(\omega)$, only combinations such as $\mu_{\text {pair }} / \gamma_{2}$ or $m \gamma_{2}$ appear in low frequency $(\ll \Omega)$ observables.

33 This formula assumes that $\gamma_{2} \Omega<\mathcal{E}_{a}$ and $\widetilde{\mathcal{E}}_{c} \ll T$. For larger values of $\Omega$, the first term still increases with the ratio $\Omega / \mathcal{E}_{a}$, but slower than $\Omega /\left(4 \pi \mathcal{E}_{a}\right)$. For $\widetilde{\mathcal{E}}_{c}$ comparable to or greater than $T$, the second term will decay slower with increasing $\widetilde{\mathcal{E}}_{c}$, and won't be zero or even negative, as this small $\widetilde{\mathcal{E}}_{c}$ formula would suggest.

34 The pair life time is thus on the order of $1 / \widetilde{\Omega}$ at $\left(T_{c}+T^{*}\right) / 2$. If $1 / \widetilde{\Omega} \ll 1 / T$, as seems to be the case according to our analysis, this implies that bosonic transport is negligible well before $T^{*}$ is reached, as is confirmed by all transport experiments including the Nernst effect.

35 We write $T_{c}=\max \left(1.6 T^{*}-0.5 T^{* 2}-0.18,0\right)$ in units of roughly $100 \mathrm{~K}$, to semiquantitatively fit the phase diagram of hole-doped cuprates. For all the $2 \mathrm{D}$ systems, we take $\widetilde{\mathcal{E}}_{c} \approx 0.05 \mathrm{~K}$, while for the $3 \mathrm{D}$ system illustrated in the insets of Fig. 4 we take $\widetilde{\mathcal{E}}_{c} \approx 15 \mathrm{~K}$. These are chosen to be consistent with the observed 3D-2D crossover temperatures of various materials. 